\title{
Obeso metabólicamente normal
}

Metabolically healthy obese patients

\author{
Jaime Pajuelo R. ${ }^{1}$, Hugo Arbañil H. ${ }^{2}$, José Sánchez Gonzales ${ }^{2}$, Dante Gamarra G. ${ }^{2}$, \\ Harold Torres A. ${ }^{2}$, Rosa Pando ${ }^{1,2}$, Rosa Agüero Z. ${ }^{2}$ \\ ${ }^{1}$ Instituto de Investigaciones Clínicas, Facultad de Medicina, Universidad Nacional Mayor de San Marcos, Lima, Perú. \\ ${ }^{2}$ Servicio de Endocrinología, Hospital Nacional Dos de Mayo, Lima, Perú.
}

\begin{abstract}
Resumen
Introducción: La obesidad es un problema de Salud Pública que tiende a incrementarse y que compromete la salud de las personas. Objetivos: Conocer la prevalencia de obesos metabólicamente normales (OMN) en función de la presencia de factores de riesgo cardiovascular en una población adulta que presenta obesidad y obesidad mórbida Diseño: Estudio observacional, descriptivo y de corte transversal. Lugar: Servicio de Endocrinología, Hospital Dos de Mayo, Lima. Perú Participantes: Personas adultas sin enfermedades metabólicas. Intervenciones: En el período abril de 2009 a mayo de 2010, se estudió a todas aquellas personas adultas que consultaron por obesidad y que dijeron no tener diabetes mellitus tipo 2, hipertensión arterial y dislipidemias. Se les pesó, talló y se midió su circunferencia de cintura (CC). Con las dos primeras se calculó el índice de masa corporal y quienes tenían $30 \mathrm{~kg} / \mathrm{m} 2$ o más ingresaron al estudio. Se les midió insulina, glucosa y perfil lipídico. Con la glucosa, c-HDL, triglicéridos y la CC se determinó el síndrome metabólico y con la insulina y la glucosa, el modelo homeostático de evaluación de la resistencia en insulina (HOMA-IR), con la finalidad de identificar resistencia a la insulina (RI). Se dividió el grupo en dos: obesos de 30 a $39,9 \mathrm{~kg} / \mathrm{m} 2$ y $40 \mathrm{~kg} / \mathrm{m} 2$ y más. El análisis estadístico se efectuó con las pruebas de t-student y de chi-cuadrado, con un intervalo de confianza de $95 \%$ y nivel de significancia $<0,05$. Principales medidas de resultados: Promedio y desviación estándar de las variables estudiadas y prevalencia de OMN y OMnoN. Resultados: La población estudiada alcanzó a 158 personas, de las cuales 91,2\% correspondió al género femenino. Entre las diferencias entre los dos grupos $O M N$ y $O M n o N$, referente a las variables antropométricas y bioquímicas tuvieron significación estadística el peso, talla, CC, insulina y el HOMA-IR. La prevalencia de OMN, de acuerdo a Wildman, fue $7 \%$ en el total de obesos y 7,3 y $6,4 \%$ en los grupos OMN y OMnoN, mientras según los criterios de Meiggs, fue 13\%, 15,5 y 2,1\%, respectivamente. Conclusiones: El estudio mostró la presencia de un reducido número de obesos que fueron caracterizados como OMN.
\end{abstract}

Palabras clave: Obeso metabólicamente normal, obesidad, riesgo cardiovascular.

\section{Abstract}

Introduction: Obesity is a public health problem that tends to increase and compromises the health of the people. Objectives: To determine the prevalence of metabolically normal obese $(\mathrm{OMN})$ subjects depending on the presence of cardiovascular risk factors in an adult population showing obesity and morbid obesity. Design: Observational, descriptive and cross-section study. Setting: Department of Endocrinology, Hospital Dos de Mayo, Lima, Peru. Participants: Obese adults without metabolic diseases. Interventions: The study was conducted during the period between April 2009 and May 2010 in all adults consulting for obesity and who declared not having type 2 diabetes mellitus, arterial hypertension or dyslipidemia. Weight, height and waist circumference (WC) were obtained, and body mass index (BMI) was calculated. Those who had $30 \mathrm{~kg} / \mathrm{m} 2$ or more were included in the study. Insulin, glucose and lipid profile were determined. Metabolic syndrome was determined through measurement of glucose, HDL-C, triglycerides and WC. Insulin resistance $(\mathrm{IR})$ was diagnosed by glucose and insulin resistance homeostasis model assessment (HOMA-IR). Two groups were distinguished: obese with BMI of 30 to $39.9 \mathrm{~kg} / \mathrm{m} 2$, and obese with BMl of $40 \mathrm{~kg} / \mathrm{m} 2$ and more. Statistical analysis was performed using student $t$ test and chi -square with a $95 \%$ confidence interval and a significance level of $<0.05$. Main outcome measures: Average and standard deviation variables; prevalence of OMN and OMnotN. Results: From the 158 subjects, $91.2 \%$ were female. The OMN and OMnotN groups showed statistically significant difference in weight, height, WC, insulin and HOMA-IR. OMN prevalence bt Wildman criteria was $7 \%$ in all obese and 7.3 and $6.4 \%$ in the OMN and OMnotN groups, while by Meiggs criteria it was 13\%, 15.5 and $2.1 \%$ respectively. Conclusions: By the criteria used the study showed the presence of a reduced number of obese subjects considered OMN.

Keywords: Metabolically normal obese, obesity, cardiovascular risk.

An Fac med. 2014;75(2):113-8 / doi: http://dx.doi.org/10.15381/anales.v75i2.8386 


\section{INTRODUCCIÓN}

Los procesos que se vienen dando en el mundo, como consecuencia de la globalización, han traído una serie de cambios tanto positivos como negativos. Dentro de los primeros tenemos la disminución de la mortalidad infantil, la mejora en la tasa global de fecundidad, el aumento de la expectativa de vida, entre otros; y dentro de los negativos, la aparición de las enfermedades emergentes, como son la obesidad, diabetes mellitus tipo 2, hipertensión arterial, dislipidemias y algunos cánceres.

Se estima que el número personas con obesidad en el mundo sobrepasa los 300 millones ${ }^{(1)}$. Las consecuencias de la obesidad son dramáticas y se dice que el riesgo de muerte súbita en los obesos es tres veces más que en los no obesos, y es el doble para el desarrollo de insuficiencia cardíaca congestiva (ICC), enfermedad coronaria vascular (ECV) y cardiopatía isquémica, mientras que la posibilidad de desarrollar diabetes mellitus tipo 2 es 93 veces mayor cuando el índice de masa corporal (IMC) pasa de $35 \mathrm{~kg} / \mathrm{m}^{2}{ }^{(2)}$. El riesgo de desarrollar complicaciones en la obesidad es proporcional al grado de obesidad y más específicamente al incremento de la grasa visceral ${ }^{(3)}$.

En los estudios con representación nacional, realizados en el Perú, se ha mostrado que la prevalencia de sobrepeso ha pasado de 24,9 a $32,6 \%$ y la de obesidad de 9 a 14,2\%, en el período de 1975 al $2005^{(4,5)}$. Si bien las prevalencias han aumentado, lo más dramático es cuando se infiere a la población adulta de esos años, de 2374780 de adultos con sobrepeso y obesidad para el año 1975 se ha incrementado a 7724829 al año $2005^{(6)}$.

El hecho de ser obeso conlleva a que presente mayores prevalencias de síndrome metabólico (SM) ${ }^{(7)}$, resistencia a la insulina (RI) ${ }^{(8)}$ y dislipidemias ${ }^{(9)}$. Sin embargo, existen algunos obesos que no presentan estas complicaciones, ya que pareciera que están protegidos de las anormalidades metabólicas ${ }^{(10)}$. Ya Keyes ${ }^{(11)}$, en 1973, y posteriormente
Andres ${ }^{(12)}$, en 1980, sugirieron que la obesidad para algunos no representa un riesgo; muy por el contrario, viene a ser una ventaja. Sims, en una amplia revisión, concluyó que hay demasiadas evidencias de la presencia de individuos obesos que sin embargo son metabólicamente normales ${ }^{(13)}$.

Estos individuos son sensibles a la insulina y tienen un perfil cardiovascular favorable, pese a su exceso de grasa corporal ${ }^{(13)}$. Aproximadamente 10 a $25 \%$ de los obesos y una muy pequeña fracción de obesos mórbidos no se encuentran afectados por alteración metabólica alguna ${ }^{(14)}$. Wildman ya encontró prevalencia alta de personas con IMC normal y anormalidades metabólicas; y, de la misma manera, personas obesas sin la presencia de esta anormalidades ${ }^{(15)}$.

El objetivo del presente estudio fue conocer la prevalencia de obesos metabólicamente normales (OMN) en función de la presencia de factores de riesgo cardiovascular en una población adulta que presentaba obesidad y obesidad mórbida, atendida en el Servicio de Endocrinología del Hospital Dos de Mayo.

\section{MÉTODOS}

El estudio fue observacional, descriptivo y de corte transversal, realizado en el Servicio de Endocrinología del Hospital Nacional Dos de Mayo, Lima, Perú, en el período comprendido entre abril del año 2009 hasta marzo de 2010. Los criterios de inclusión fueron ser mayor de 20 años y consultar por aumento de peso; y los de exclusión fueron no tener diabetes mellitus, hipertensión arterial o dislipidemias al momento de la consulta, ni ser portador de alguna enfermedad que distorsionara el peso o la talla o ambas a la vez. La población estudiada alcanzó a 158 personas, de las cuales $91,2 \%$ correspondió al género femenino.

A todos se los pesó y talló de acuerdo a la metodología aceptada internacionalmente ${ }^{(16)}$. Con ambas medidas se calculó el índice de masa corporal (IMC) expresado en kilogramos/metro ${ }^{2}$. La circunferencia de la cintura (CC) fue medida en el punto medio de la línea axilar, de la distancia que va entre el reborde inferior de la última costilla y la cresta ilíaca ${ }^{(17)}$. El diagnóstico de obesidad se hizo de acuerdo a la clasificación de la Organización Mundial de la Salud (OMS) (18) y con el siguiente criterio, mayor o igual a $30 \mathrm{~kg} / \mathrm{m} 2$. Para los fines del estudio, el grupo fue dividido en obesos (IMC $\geq 30$ a $39,9 \mathrm{~kg} / \mathrm{m} 2$ ) $(n=110)$ y obesos mórbidos (IMC $\geq 40$ $\mathrm{kg} / \mathrm{m} 2)(\mathrm{n}=48)$.

En sangre extraída por venopuntura se dosó colesterol total (CT) y colesterol-HDL (C-HDL) con el método enzimático colesterol oxidasa, triglicéridos ( $\mathrm{Tg})$ mediante el método enzimático glicerol quinasa y el colesterol-LDL (CLDL) con la fórmula de Friedewald. La glucosa, por el método enzimático de la glucosa oxidasa. Asimismo, la insulina basal por el método de electroquimioluminiscencia. El ayuno fue de 12 horas.

La resistencia a la insulina (RI) fue determinada mediante el HOMA IR, utilizando la ecuación de Matthews (insulina basal $(\mathrm{uUI} / \mathrm{mL})$ * glicemia basal $(\mathrm{mmol} / \mathrm{L}) / 22,5^{(19)}$. El criterio diagnóstico para identificar las personas con RI fue un HOMA IR de $>=2,7^{(20)}$.

Para el diagnóstico de SM se ha trabajado con los criterios dados por el ATP III ${ }^{(21)}$, y estos son: $C C \geq 102 \mathrm{~cm}$ $y \geq 88 \mathrm{~cm}$, para varones y mujeres, respectivamente, $\mathrm{TG} \geq 150 \mathrm{mg}$, C-HDL $\leq 40 \mathrm{mg}$ en varones y $\leq 50 \mathrm{mg}$ en la mujer y glicemia $\geq 110 \mathrm{mg}$. La presión arterial no fue considerada.

Los criterios para definir dislipidemia fueron: CT $\geq 200 \mathrm{mg}$, TG $\geq 150 \mathrm{mg}$, C$\mathrm{HDL} \leq 40 \mathrm{mg}$ para varones y $\leq 50 \mathrm{mg}$ en mujeres y C-LDL $\geq 160 \mathrm{mg}{ }^{(21)}$.

Para la glicemia se usó el valor de $\geq 100 \mathrm{mg}$.

Para la definición de obeso metabólicamente normal, se empleó los criterios de Meiggs ${ }^{(22)}$, que se refieren a la no presencia de RI, y los de Wildman ${ }^{(15)}$ 
que mencionan que un obeso es metabólicamente normal si solo tiene la presencia de un factor de riesgo; estos factores son elegidos en forma arbitraria. Para el caso del presente estudio se ha elegido SM, RI, CT, C-HDL, C-LDL, TG y glicemia.

Para definir OMS, se empleó los criterios de Wildman ${ }^{(15)}$ y de Meiggs ${ }^{(22)}$, que fueron modificados para el presente estudio. En el caso de Wildman, empleó las variables de presión arterial, niveles de Tg, C-HDL, glicemia, RI e indicadores de inflamación. En nuestro caso, se usó niveles de Tg, C-HDL, glicemia y RI, adicionándose SM, CT, y C-LDL. El criterio de Meiggs era solo la presencia de RI, pero se le agregó el SM.

Para el análisis, se ha utilizado el paquete estadístico SPSS 17.0. Los resultados son presentados en medida de tendencia central, de dispersión. Se usó las prueba t-student y para las variables categóricas, la de chi cuadrado, con un valor de significación estadística de $<0,05$.

\section{RESULTADOS}

En la tabla 1 se puede observar que predominó el grupo femenino $(91,2 \%)$ frente al masculino. El promedio de edad de los obesos mórbidos era mayor (46,6 años); asimismo, en lo que se refiere a las variables antropométricas, todos sus promedios fueron superiores, con excepción de la talla. Las diferencias del peso, IMC y CC fueron estadísticamente significativas $(\mathrm{p}<0,001)$.

En cuanto a las variables bioquímicas, existió la misma tendencia, a excepción de los triglicéridos, siendo las únicas con significación estadística la insulina $(p<0,001)$ y el HOMA-I $(\mathrm{p}<0,001)$.

En la tabla 2 se puede observar el número, porcentaje e intervalos de confianza al $95 \%$ de los obesos que presentaban riesgos cardiovasculares. Los riesgos considerados fueron la $\mathrm{RI}, \mathrm{CT}$, C-HDL, C-LDL, TG, glucosa y SM. El 3,2\% del total de obesos tenía los 7 ries-
Tabla 1. Porcentaje, promedio, desvío estándar (DE) y significación estadística ( $p$ ) de las variables estudiadas entre los dos grupos

\begin{tabular}{ccccc} 
& $\begin{array}{c}\text { Total } \\
(158)\end{array}$ & $\begin{array}{c}\text { Obeso } \\
(\mathrm{n}=110)\end{array}$ & $\begin{array}{c}\text { Obeso mórbido } \\
(\mathrm{n}=48)\end{array}$ & $p$ \\
\hline Género $(8,8 \%)$ & $9(\%)$ & $8,3(\%)$ & \\
& $\mathrm{F}(91,2 \%)$ & $91(\%)$ & $91,7(\%)$ & \\
\hline Edad (años) & $44,3(121)$ & $43,3(12,8)$ & $46,6(11,8)$ & 0,800 \\
\hline Peso (kg) & $87,5(14,2)$ & $81,6(11,2)$ & $101,3(12,5)$ & $<0,001$ \\
Talla (m) & $1,52(0,07)$ & $1,53(0,07)$ & $1,51(0,07)$ & 0,93 \\
IMC (kg/m²) & $37,8(5,7)$ & $34,8(3,3)$ & $44,5(3,8)$ & $<0,001$ \\
CC (cm) & $109,7(11,9)$ & $105,6(9,3)$ & $119,1(12,2)$ & $<0,001$ \\
\hline Glucosa (mg/dL) & $93,9(14,5)$ & $92,8(15,3)$ & $96,5(12,3)$ & 0,13 \\
Insulina (uUl/mL) & $21,2(13,2)$ & $19,1(11,7)$ & $26(15,3)$ & $<0,001$ \\
HOMA-I & $5(3,5)$ & $4,42(3)$ & $6,3(4,2)$ & $<0,001$ \\
CT(mg/dL) & $199,7(36,9)$ & $198,9(38,5)$ & $201,5(33,5)$ & 0,67 \\
C-HDL(mg/dL) & $42,1(9,7)$ & $41,9(10,1)$ & $42,3(8,7)$ & 0,8 \\
C-LDL (mg/dL) & $115,5(37,8)$ & $113,1(38,4)$ & $120,9(36,2)$ & 0,27 \\
TG (mg/dL) & $176,8(81,7)$ & $180,8(87,3)$ & $167,6(67,1)$ & 0,35 \\
\hline
\end{tabular}

gos, siendo el grupo de obesos morbidos el más comprometido, con 6,3\%. Por otro lado, existieron $1,9 \%, 1,8 \%$ y $2,1 \%$ sin riesgo alguno, respectivamente. Un $7 \%$ presentó menos de dos riesgos en el total y $7,3 \%$ en los obesos y $6,4 \%$ en los mórbidos, siendo todos estos considerados como OMN.

La tabla de contingencia permite cruzar dos variables categóricas con la finalidad de determinar si existe asociación entre ellas; estas variables son SM y RI. La tabla 3 muestra que 13\% del total y $15,5 \%$ y $2,1 \%$ de los grupos en estudio no presentaron SM ni RI. El $40,8 \%$, 39,1\% y $50 \%$ tenían SM y RI.
La presencia de SM solo ocurrió en $53,2 \%, 52,7 \%$ y $60,4 \%$, mientras que la RI en 74,5\%, 70,9\% y 87,5\%. Los valores de chi-cuadrado permiten afirmar que estas variables son independientes, no existiendo asociación entre ellas.

\section{DISCUSIÓN}

Tradicionalmente, se ha relacionado a la obesidad con una serie de complicaciones que van a dañar la salud e incluso poner en peligro la vida de las personas; esto es completamente cierto y comprobado. Sin embargo, nuestros
Tabla 2. Número y porcentaje de riesgos cardiovasculares, en el total y grupos de obesos.

\begin{tabular}{|c|c|c|c|c|c|c|c|c|c|}
\hline \multirow{2}{*}{ Riesgos } & \multicolumn{3}{|c|}{ Total } & \multicolumn{3}{|c|}{ Obeso } & \multicolumn{3}{|c|}{ Obeso mórbido } \\
\hline & $\mathrm{n}$ & $\%$ & Ic95\% & $\mathrm{n}$ & $\%$ & Ic95\% & $\mathrm{n}$ & $\%$ & Ic95\% \\
\hline 7 & 5 & 3,2 & $1,5-4,8$ & 2 & 1,8 & $0-3$ & 3 & $6-3$ & $4-8-5$ \\
\hline 6 & 29 & 18,4 & $14,6-22,1$ & 20 & 18,2 & $14,4-21,9$ & 9 & 18,8 & $15-22,6$ \\
\hline 5 & 44 & 27,8 & $23,5-32,1$ & 30 & 27,3 & $22,9-31,6$ & 14 & 29,2 & $24,8-33,6$ \\
\hline 4 & 21 & 13,3 & $10-16,6$ & 14 & 12,7 & $9,4-15,9$ & 7 & 14,6 & $11,1-18$ \\
\hline 3 & 16 & 10,1 & $7,2-13$ & 11 & 10 & $7-12,9$ & 5 & 10,4 & $7,4-13,3$ \\
\hline 2 & 32 & 20,3 & $16,4-24,2$ & 25 & 22,7 & $18,6-26,7$ & 7 & 14,6 & $11,1-18$ \\
\hline 1 & 8 & 5,1 & $2,9-7,2$ & 6 & 5,5 & $3,2-7,7$ & 2 & 4,3 & $2,3-6,2$ \\
\hline 0 & 3 & 1,9 & $0,1-3,2$ & 2 & 1,8 & $0,0-3$ & 1 & 2,1 & $0,1-3,4$ \\
\hline
\end{tabular}


Tabla 3. Tabla de contingencia $2 \times 2$, entre sindrome metabólico (SM) y resistencia a la insulina (RI), en el total y grupos de obesos.

\begin{tabular}{cccc} 
& Total & Obeso & Obeso mórbido \\
SM+RI & 40,8 & 39,1 & 50 \\
SM no RI & 12,4 & 13,6 & 10,4 \\
RI no SM & 33,7 & 31,8 & 37,5 \\
No RI no SM & 13 & 15,5 & 2,1 \\
\hline & $X^{2} 0,730$ & $X^{2} 0,706$ & $X^{2} 0,923$ \\
\hline
\end{tabular}

estudios (7-9), han mostrado que existe un gran porcentaje de obesos que no presenta estos riesgos

A nivel nacional se ha comunicado que conforme se incrementa el IMC las prevalencias de hiperglicemia, de dislipidemias (CT, C-HDL, C-LDL y Tg) y de presión arterial (sistólica y diastólica) también siguen la misma tendencia ${ }^{23)}$. Lo mismo ha sido encontrado en otros estudios referente a RI y la hiperinsulinemia ${ }^{(24,25)}$.

Comparando los resultados del presente estudio con los de algunos países de Europa (Islandia, España e Irlanda), donde la población estudiada fue obesa y de 20 a 39 años, vemos que nuestros niveles de CT son más o menos similares. Caso contrario sucede con los Tg, el C-HDL, la insulina y el HOMA-I, que son más elevados, lo que representa de alguna manera mayor riesgo cardiovascular ${ }^{(26)}$.

La obesidad ya implica un riesgo. Sin embargo, existe una gran cantidad de personas que no presentan estos riesgos y a quienes se los ha rotulado como obesos metabólicamente normales $(\mathrm{OMN})$. Si bien este término genera mucha discusión de tipo conceptual, nosotros lo aceptamos en el entendido que un $\mathrm{OMN}$ es aquel que no presenta complicaciones metabólicas. Estos individuos, a pesar de tener un exceso de grasa corporal, tienen un perfil metabólico caracterizado por triglicéridos, C-HDL, glucosa, HOMA-I en niveles favorables para su salud, sugiriendo que esto podría deberse a la diferenciación de la acumulación de la grasa visceral, al peso al nacer, al tamaño de las células adiposas y a factores genéticos ${ }^{(13)}$. En ese sentido, en el GWAS (genoma wide association study) se identificó un locus cerca IRS1 que está asociado significativamente con el porcentaje de grasa corporal y que el incremento de este alelo presenta una fuerte asociación con un perfil metabólico favorable ${ }^{(27)}$.

Otra de las razones por las cuales el OMN tiene esas ventajas es debido a su perfil inflamatorio. El trabajo de Van Guilder demostró que en los obesos con SM existe una mayor presencia de marcadores biológicos de estrés oxidativo e inflamación, como la LDL oxidada, el factor de necrosis tumoral, la interleuquina 6 y 8 y la proteína C-reactiva, frente a los otros obesos sin SM, siendo estas diferencias estadísticamente significativas. En el mismo trabajo se señala que estas diferencias también ocurren con la resistencia a la insulina (HOMA-I) ${ }^{(28)}$. Karelis demostró que los OMN presentan un perfil inflamatorio favorable, con niveles menores de proteína C-reactiva y que esto podría estar asociado a tener menor riesgo a las complicaciones cardiovasculares ${ }^{(29)}$. El CoLaus Study, utilizando varios criterios para definir OMN, encontró niveles bajos de proteína $C$-reactiva y que de alguna manera esto sería un elemento importante en la 'protección' de este grupo ${ }^{(30)}$.

Existen muchos criterios para poder identificar a un $\mathrm{OMN}$, lo que hace muy dificultoso el hacer comparaciones. Dentro de toda esta variedad de criterios están los de Wildman ${ }^{(15)}$ (elevación de presión arterial, de Tg y de glucosa, disminución de C-HDL y RI, y presencia de marcadores inflamatorios), defi- niendo como $\mathrm{OMN}$ a aquellas personas que presentan menos de dos riesgos. Wildman encontró un 9.7\% de OMN. En nuestro estudio hemos optado por este criterio, ya que es lo más parecido a los riesgos utilizados. En ese sentido, existió un $7 \%$ de OMN, con mayor presencia en el obeso $(7,3 \%)$ que en el obeso mórbido (6,4\%). Si bien hay una menor prevalencia, no se puede comparar, por cuanto las definiciones son parecidas pero no iguales; el estudio de Wildman fue en una población general.

De todas maneras, llama la atención que tanto en el grupo obeso como en el obeso mórbido existan personas que presentan todos los riesgos frente a otros que no tienen alguno; incluso hay $2,1 \%$ de obesos mórbidos con estas características. En lo que respecta al género todas, son del grupo femenino y esto responde a que la mayoría de personas en el estudio fueron mujeres. Por otro lado, dentro de los dos riesgos con mayor presencia estuvieron la RI y el C-HDL bajo y en contraposición se encontró la glicemia. Si bien la población estudiada fue pequeña, llama mucho la atención que teniendo un mismo IMC la posibilidad de enfrentar un problema cardiovascular a futuro sea diferente. La edad promedio en ambos grupos no alcanzó los 50 años. Lamentablemente no se sabe desde cuándo eran obesos.

Sin embargo, el estudio Pizarra ${ }^{(31)}$ concluye que, con el tiempo (6 y 11 años), algunos de los que fueron $\mathrm{OMN}$ dejaron de serlo y que además el riesgo de desarrollar DM se incrementó de la misma manera que en el grupo de obesos metabólicamente no sanos, y por estas razones mencionan que el concepto de OMN debe ser considerado como un proceso dinámico. La comparación de OMN con personas de un IMC normal y sin la presencia de riesgos ha mostrado que, en función del tiempo (7 años), la aparición de complicaciones se hace con mayor incidencia en el primer grupo; pero, es de destacar que pese al tiempo hay algunos que continúan con su condición inicial ${ }^{(32)}$. En este sentido, debería hacerse estudios de seguimien- 
to para ver en qué momento y en qué condiciones dejan de serlo o no.

Es probable que la $\mathrm{OMN}$ sea un estado de riesgo bajo a intermedio en lugar de permanente. En apoyo de esta hipótesis, datos recientes del North West Adelaide Health Study sugieren que la OMN sería un fenotipo transitorio en una cierta proporción de individuos. De todos los individuos clasificados como OMN al inicio del estudio, la tercera parte viró hacia un fenotipo de riesgo alto durante el curso del mismo, pero el menor riesgo de diabetes tipo 2 y de enfermedad cardiovascular se limitó al subgrupo de OMN que mantuvo esta condición ${ }^{(33)}$.

Otra de las definiciones usadas fue la propuesta por Meiggs (22), quien opta por un solo criterio y es referido a la presencia o no de RI. En el caso del estudio, hemos sido más exigentes y le hemos adicionado el SM, siendo OMN aquellos que no presentaban SM o RI. En ese sentido, la prevalencia comunicada de OMN es $13 \%$ en el total, con $15,5 \%$ en los obesos y $2,1 \%$ en los obesos mórbidos. En contraposición, las prevalencias de los que tuvieron a la vez SM y RI fueron 40,8\%, 39,1\% y $50 \%$, respectivamente, lo que nos dice que los que se encuentran con mayor riesgo son los obesos mórbidos. Para los que tienen alguna objeción por el uso del IMC para definir obesidad, Shea demostró la ausencia de complicaciones metabólicas en personas obesas diagnosticadas por medio del IMC, pero también por el porcentaje de grasa corporal mediante absorciometría de energía dual de rayos $\mathrm{X}^{(34)}$.

Algunos estudios han realizado sus definiciones con SM y RI, pero en forma separada. Así tenemos el estudio de Cremona ${ }^{(35)}$, en el que se encontró $11 \%$ de OMN; la identificación de los mismos se hizo solo con la presencia o no de RI. En el de Iacobelli se encontró $27,5 \%$ y basó su identificación en la presencia o no de SM ${ }^{(36)}$. Nuestro estudio presenta prevalencias de 40,8\% para personas con $\mathrm{SM}+\mathrm{RI}$, de $12,4 \%$ con SM y de $33,7 \%$ con RI.
Los resultados divergentes se explicarían por el empleo de diferentes definiciones y por los diferentes puntos de corte para definir la salud metabólica, al emplear el HOMA-IR y los otros factores de riesgo, lo que trae como consecuencia una gran confusión al comparar los trabajos. Estas razones han llevado a Messier sugerir la necesidad de un consenso que sirva para estandarizar esta definición ${ }^{(37)}$.

En conclusión, el estudio mostró la presencia de un reducido número de obesos, que se caracterizaron por ser considerados OMN, de acuerdo a los criterios utilizados.

\section{REFERENCIAS BIBLIOGRÁFICAS}

1. Rossner S. Obesity, the disease of the 21 th century. Int J Obes. 2002;26:S2-S4. doi: doi: http://dx.doi. org/10.1038/sj.ijo.0802209

2. Racetta S, Dusinger S, Deusinger R. Obesity: overview of prevalence, etiology and treatment. Phys Ther. 2003;83:276-88.

3. Bluher M. The distinction of metabolically 'healthy' from 'unhealthy' obese individuals. Curr Opin Lipidol. 2010;21:38-42. doi: 10.1097/ MOL.0b013e3283346ccc. doi: http://dx.doi. org/10.1097/MOL.0b013e3283346ccc

4. Pajuelo J. Estado nutricional del adulto en el Perú. Acta Méd Peruana. 1992;16:22-32.

5. Instituto Nacional de Salud (IMS). Centro Naciona de Alimentación y Nutrición (CENAN). Encuesta nacional de indicadores nutricionales, bioquímicos, socioeconómicos y culturales. Lima 2005.

6. Pajuelo J. El sobrepeso y la obesidad en el Perú: un problema a enfrentar. Facultad de Medicina. UNMSM. Lima 2012.

7. Pajuelo J, Sánchez Abanto J. El sindrome metabólico en el Perú. An Fac med. 2007;68(1):38-46.

8. Pajuelo J. Resistencia a la insulina en mujeres con sobrepeso y obesidad de acuerdo a su grasa visceral. Tesis para optar el título de Magister en Nutrición, mención en Nutrición Clínica. Facultad de Medicina UNMSM. Lima 2006.

9. Pajuelo J. Los lípidos en adolescentes con sobrepeso y obesidad. $X$ Congreso Nacional de Alimentación y Nutrición. Sociedad Peruana de Nutrición. Lima 2011.

10. Primeau V, Coderre L, Karelis A, Brochu M, Lavoie M, Messier V, Sladek R, Rabasa-Lloret R. Characterizing the profile of obese patients who are metabolically healthy. Int J Obes. 2011;35:971-81. doi: http://dx.doi.org/10.1038/ijo.2010.216

11. Keyes A. Overweight and the risk of sudden heart attack and sudden death. En: Obesity in Perspective. DHEW Publication. 1973:75-708.

12. Andres R. Effect of obesity on total mortality. Int $\mathrm{J}$ Obes. 1980;4:381-6.

13. Sims E. Are there persons who are obese, but metabolically healthy? Metabolism. 2001;50:1499-504. doi: http://dx.doi.org/10.1053/meta.2001.27213

14. Soverini V, Moscatiello S, Villanova N, Ragni E, Di Domizio S, Marchesini G. Metabolic syndrome and insulin resistance in subjects with morbid obesity. Obes Surg. 2010;20:295-301. doi: http://dx.doi. org/10.1007/s1 1695-009-9999-Z

15. Wildman RP, Muntner P, Reynolds K, McGinn AP, Rajpathak S, et al. The obese without cardiometabolic risk factor clustering and the normal weight with cardiometabolic risk factor clustering: prevalence and correlates of 2 phenotypes among the US population (NHANES 1999-2004). Arch Intern Med. 2008;168(15):1617-24. doi: http://dx.doi. org/10.1001/archinte.168.15.1617

16. Lohman T, Roche A, Martorell R. Anthropometric Standardization Reference Manual. Human Kinetics Books. 1988.

17. Wang J, Thornton J, Bari S, Williamson B, Gallagher $\mathrm{D}$, Heymsfield $\mathrm{S}$, et al. Comparisons of waist circumferences measured at 4 sites. Am J Clin Nutr 2003;77(2):379-84.

18. World Health Organization (WHO). Obesity: preventing and managing the global epidemic. Geneva 2000.

19. Matthews DR, Hosker JP, Rudenski AS, Naylor BA, Teacher DF, Turner RC. Homeostasis model assessment: insulin resistance and beta-cell function from fasting plasma glucose and insulin concentrations in man. Diabetologia. 1985;28(7):412-9. doi: http://dx.doi.org/10.1007/BF00280883

20. Bonora E, Kiechi S, Willeit J. Prevalence of insulin resistance in metabolic disorders. The Bruneck Study. Diabetes. 1998;47:1643-9. doi: http://dx.doi. org/10.2337/diabetes.47.10.1643

21. Expert Panel on Detection, Evaluation and Treatment of High Blood Cholesterol in Adults: Executive summary of the Third Report of the National Cholesterol Education Program (NCEP) Expert Panel Detection, Evaluation and Treatment of High Blood Cholesterol in Adults (Adult Treatment Panel III). JAMA. 2001;285:2486-97. doi: http://dx.doi. org/10.1001/jama.285.19.2486

22. Meigs J, Wilson P, Fox C, Vasan R, Nathan D, Sullivan $\mathrm{L}$, et al. Body mass index, metabolic syndrome and risk of type 2 diabetes or cardiovascular disease. J Clin Endocrinol Metab. 2006;91:2006-12. doi: http://dx.doi.org/10.1210/jc.2006-0594

23. Pajuelo J, Sánchez Abanto J. Estado nutricional del adulto en relación al riesgo cardiovascular. Rev Soc Peru Med Interna. 2010;23(3):85-91.

24. Fernández R, Arrauz M, Ortega J, Hernández J. Resistencia a la insulina y cambios metabólicos en adultos obesos. Rev Cubana Endocrinol. 2011;22(2):13-8.

25. Rogero ME, Alba-il MR, Sánchez M, Rabanal A, Olivas A, Garcia C. Prevalencia de resistencia a la insulina en una población de adultos jóvenes. Relación con el estado ponderal. Endocrinol Nutr. 2012;59(2):98-104. doi: http://dx.doi.org/10.1016/j. endonu.2011.10.009

26. Ramel A, Halldorsson TI, Tryggvadottir EA, Martinez JA, Kiely M, Bandarra NM, Thorsdottir I. Relationship between BMI and body fatness in three European countries. Eur J Clin Nutr. 2013;67:254-8. doi: http://dx.doi.org/10.1038/ejcn.2013.6

27. Kilpeläinen TO, Zillikens MC, Stančákova $A$, Finucane FM, Ried JS, Langenberg C, et al. Genetic variation near IRS1 associates with reduced adiposity and an impaired metabolic profile. Nut Genet 2011;43:753-760. doi: http://dx.doi. org/10.1038/ng.866

28. Van Guilder G, Hoetzer G, Greiner J, Stanfer B, De Souza C.. Influence of metabolic syndrome on biomarkers of oxidative stress and inflammation in obese adults. Obesity. 2006;14(12):2127-31. doi: http://dx.doi.org/10.1038/oby.2006.248 
29. Karelis A, Faraj M, Bastard JP, St-Pierre D, Brochu M, Prud'home, Rabasa-Lloret R. The metabolically healthy obese individual presents a favorable inflammation profile. J Clin Endocrinol Metab. 2005;90:4145-50. doi: http://dx.doi.org/10.1210/ jc. 2005-0482

30. Marques-Vidal P, Velho S, Waterworth D, Waeber G, von Kanel R, Vollenweider P. Association between inflammatory biomarkers and metabolically healthy obesity depends of the definition used. Eu J Clin Nutr. 2012;66(4):426-35. doi: http://dx.doi. org/10.1038/ejcn.2011.170

31. Soriguer F, Gutiérrez-Repiso C, Rubio-Martin E, Garcia-Fuentes E, Almaraz MC, et al. metabolicaIly healthy but obese, a matter of time? Findings from the prospective Pizarra Study. J Clin Endocrinol Metab. 2013;98:2318-25. doi: http://dx.doi. org/10.1210/jc.2012-4253

32. Chang Y, Ryu S, Suh B, Yun K, Kim C, Cho S. Impact of $\mathrm{BMI}$ on the incidence of metabolic abnor- malities in metabolically heatlthy men. Int J Obes. 2012;36:1187-94. doi: http://dx.doi.org/10.1038/ ijo.2011.247

33. Stefan N, Haring H, Hu F, Schulze M. Metabolically healthy obesity: epidemiology mechanism and clinical implications. Lancet Diabetes Endocrinol. 2013;1(2):152-61. doi: http://dx.doi.org/10.1016/ S2213-8587(13)70062-7

34. Shea J, Randell E, Sun G. The prevalence of metabolically healthy obese subjects defined by BMI and dual-energy X-ray absorptiometry. Obesity. 2011;19(3):624-30. doi: http://dx.doi.org/10.1038/ oby. 2010.174

35. Calori G, Lattuada G, Piemonti L, Garancini MP, Ragogna F, Villa M, et al. Prevalence, metabolic features, and prognosis of metabolically healthy obese Italian individuals. Diabetes Care. 2011;34:210-5. doi: http://dx.doi.org/10.2337/dc10-0665

36. lacobelli G, Ribaudo M, Zappaterreno A, Iannucci C, Leonetti F. Prevalence of uncomplicated obesity in a Italian obese population. Obes Res. 2005;13:116-22.

37. Messier V, Karelis A, Prud'homme D, Brochu M, Rabasa-LLoret R. Identifying metabolically healthy but obese individuals in sedentary postmenopausal women. Obesity. 2010;18:911-7. doi: http://dx.doi. org/10.1038/oby.2009.364

Artículo recibido el 18 de diciembre de 2013 y aceptado para publicación el 13 de enero de 2014.

El autor declara no existir conflictos de interés.

Correspondencia:

Dr. Jaime Renato Pajuelo Ramírez

Correo electrónico: japara18@yahoo.com 